\title{
A Numerical Approach for Singularly Perturbed Nonlinear Delay Differential Equations Using a Trigonometric Spline
}

\author{
M. Lalu ${ }^{1}{ }^{1}$ and K. Phaneendra ${ }^{2}{ }^{2}$ \\ ${ }^{1}$ Department of Mathematics, University College of Science, Osmania University, Hyderabad, India \\ ${ }^{2}$ Department of Mathematics, University College of Engineering, Osmania University, Hyderabad, India \\ Correspondence should be addressed to K. Phaneendra; kollojuphaneendra@yahoo.co.in
}

Received 15 December 2021; Revised 6 January 2022; Accepted 26 January 2022; Published 13 February 2022

Academic Editor: Qichun Zhang

Copyright (C) 2022 M. Lalu and K. Phaneendra. This is an open access article distributed under the Creative Commons Attribution License, which permits unrestricted use, distribution, and reproduction in any medium, provided the original work is properly cited.

\begin{abstract}
In this paper, a computational procedure for solving singularly perturbed nonlinear delay differentiation equations (SPNDDEs) is proposed. Initially, the SPNDDE is reduced into a series of singularly perturbed linear delay differential equations (SPLDDEs) using the quasilinearization technique. A trigonometric spline approach is suggested to solve the sequence of SPLDDEs. Convergence of the method is addressed. The efficiency and applicability of the proposed method are demonstrated by the numerical examples.
\end{abstract}

\section{Introduction}

Consider a nonlinear singularly perturbed delay differential equation in the form

$$
\varepsilon \theta^{\prime \prime}=\mathscr{F}\left(s, \theta, \theta^{\prime}(s-\delta)\right) \text { on }(0,1),
$$

under the interval and boundary conditions

$$
\theta(s)=\mu(s) \text { on }-\delta \leq s \leq 0, \theta(1)=\gamma,
$$

where $0<\varepsilon \ll 1$ is a perturbation parameter and $\delta$ is a delay parameter of $o(\varepsilon)$. The solution $\theta(s)$ of Equations (1) and (2) must be continuous on $[0,1]$ and differentiable continuously on $(0,1)$. Suppose $\mathscr{F}$ is smooth function and follows the conditions

$$
\frac{\partial}{\partial w} \mathscr{F}(s, \theta, w) \geq 0, \frac{\partial}{\partial w} \mathscr{F}(s, \theta, w) \leq 0
$$

(1) $((\partial / \partial \theta)-(\partial / \partial w)) \mathscr{F}(s, \theta, w) \geq \varphi>0, \varphi$ is a positive constant

(2) The growth condition $\mathscr{F}(s, \theta, w)=O\left(|w|^{2}\right)$ as $w$ $\longrightarrow \infty$ for all $s \in[0,1]$ and all real $\theta$ and $w$.

Under the above conditions, Equations (1) and (2) have a unique solution [1] for $\delta=0$.

The proposed equation usually plays an important role in illustrating different applications, such as theory of nonpremixed combustion [2], geodynamics [3], oceanic and atmosphere circulation [4], and chemical reactions [5]. More attention has been given in the past to the computational analysis of SPDDEs [6-9]. However, motivation for the research and solution of the SPNDDE has been increasing in the last few years. These problems may have steep exponential boundary layers as a solution. Classical methods for solving such types of problems are ineffective since a boundary layer structure is present when the perturbation parameter goes to zero. For these equations, effective numerical methods should be established, the accuracy of which does not depend on $\varepsilon$. Hence, in this work, we proposed a higher 
order numerical scheme using a trigonometric spline which gives more accuracy with a smaller number of mesh points. The existence and originality of the solutions of a SPNDDE with shift were studied by Lange and Miura [10]. The authors in [11] presented a fixed-point strategy to solve a second order SPDDE. The authors in [12] assemble two methodical spectral Legendre's derivative methods to solve numerically the Lane-Emden, Bratu's, and singularly perturbed type equations. For generating numerical spectrum solutions to linear and nonlinear second-order boundary value problems, a new operational matrix approach based on shifted Legendre polynomials is introduced and studied in [13].

In [14], the authors proposed schemes with finite differences for solving the system of SPNDDE. In [15], a B-spline collocation method is constructed to solve Equations (1) and (2). In [16], the authors used shifted Legendre polynomials for studying the spectral collocation approach to solve neutral functional-differential equations with proportional delays. In [17], the Legendre spectral collocation approach is suggested by the authors for handling multipantograph delay boundary value problems. In [18], a new numerical method is proposed for solving a class of delay timefractional partial differential equations. The fractional partial differential equations are reduced into an associated system of algebraic equations that may be solved by some robust iterative solvers using the localization method, which is based on space-time collocation in some appropriate points. In [19], the authors developed a numerical technique for nonlinear singly perturbed two-point boundary value problems based on a noniterative integration method with a modest deviation argument.

The following is a concise summary of the contents of the paper. In Section 2, the approach of quasilinearization and the analysis of convergence are discussed. The continuous problem is discussed in Section 3. In Section 4, the procedure using a trigonometric spline for the solution of the problem is derived. Error estimates of the proposed scheme are discussed in Section 5. Numerical examples and computational results are shown in Section 6. Finally, the Section 7 ends with the conclusion.

\section{The Method of Quasilinearization}

Using the method of quasilinearization [20], the given nonlinear differential Equations (1) and (2) are reduced into a sequence of SPLDDEs. We take the initial approximation $\theta^{0}(s)$ which serves as a starting point for the function $\theta(s)$ in $\mathscr{F}$ and expand $\mathscr{F}\left(s, \theta(s), \theta^{\prime}(s-\delta)\right)$, around the function $\theta^{0}(s)$; we get

$$
\begin{aligned}
\mathscr{F}\left(s, \theta^{(1)}, \theta^{\prime(1)}(s-\delta)\right)= & \mathscr{F}\left(s, \theta^{(0)}, \theta^{\prime(0)}(s-\delta)\right)+\left(\theta^{(1)}-\theta^{(0)}\right)\left(\frac{\partial \mathscr{F}}{\partial \theta}\right)_{\left(s, \theta^{(0)}, \theta^{\prime}(0)\right)} \\
& +\left(\theta^{\prime^{(1)}}-\theta^{\prime^{(0)}}\right)\left(\frac{\partial \mathscr{F}}{\partial \theta^{\prime}}\right)_{\left(s, \theta^{(0)}, \theta^{(0)}\right)}+\cdots
\end{aligned}
$$

In general, we can write

$$
\begin{aligned}
\mathscr{F}\left(s, \theta^{(v+1)}, \theta^{\prime(v+1)}(s-\delta)\right)= & \mathscr{F}\left(s, \theta^{(0)}, \theta^{\prime(0)}(s-\delta)\right)+\left(\theta^{(v+1)}-\theta^{(v)}\right)\left(\frac{\partial \mathscr{F}}{\partial \theta}\right)_{\left(s, \theta^{(0)}, \theta^{\prime}(0)\right)} \\
& +\left(\theta^{\prime(v+1)}-\theta^{\prime(v)}\right)\left(\frac{\partial \mathscr{F}}{\partial \theta^{\prime}}\right)_{\left(s, \theta^{(0)}, \theta^{\prime(0)}\right)}+\cdots,
\end{aligned}
$$

for $v=0,1,2, \cdots$

Using the quasilinearization technique, Equations (1) and (2) become

$$
\begin{aligned}
& \varepsilon \theta^{\prime \prime(v+1)}(s)+\left(-\frac{\partial \mathscr{F}^{(v)}}{\partial \theta^{\prime}}\right) \theta^{\prime(v+1)}(s-\delta)+\left(-\frac{\partial \mathscr{F}^{(v)}}{\partial \theta}\right) \theta^{(v+1)}(s) \\
& =\left(\mathscr{F}^{(v)}-\left(-\frac{\partial \mathscr{F}^{(v)}}{\partial \theta}\right)-\left(\frac{\partial \mathscr{F}^{(v)}}{\partial \theta^{\prime}}\right) \theta^{\prime(v)}(s-\delta)\right), \quad v=0,1, \cdots
\end{aligned}
$$

with

$$
\theta^{(v+1)}(s)=\mu(s),-\delta \leq s \leq 0, \quad \theta^{(v+1)}(1)=\gamma,
$$

and $\mathscr{F}^{(v)}=\mathscr{F}\left(s, \theta^{(v)}, \theta^{\prime(v)}(s-\delta)\right)$. Thus, Equation (6) with Equation (7) is linear in $\theta^{(v+1)}(s)$. Now, we solve the problems given by Equations (6) and (7) using the nonpolynomial spline method.

Theoretically, the solution to the nonlinear problem satisfies

$$
\lim _{n \rightarrow \infty} \theta^{(v)}(s)=\theta^{*}(s), \quad 0 \leq s \leq 1,
$$

where $\theta^{*}(s)$ is the solution of the nonlinear problem. Computationally, we require

$$
\left|\theta^{(v+1)}(s)-\theta^{(v)}(s)\right|<\text { Tol., } \quad 0 \leq s \leq 1 .
$$

Here, Tol. is a prescribed small tolerance. Once the tolerance test is achieved, the iteration is terminated.

\section{Convergence Analysis}

The convergence of the sequence of solutions $\left\langle\theta^{(v)}\right\rangle$ is obtained as follows. For convenience purpose, we refer $\mathscr{F}(s$ $\left., \theta, \theta^{\prime}(s-\delta)\right)$ as $\mathscr{F}(\theta)$ in the entire convergence part. Consider the problem

$$
\varepsilon \theta^{\prime \prime}=\mathscr{F}(\theta)
$$

with

$$
\theta(s)=\varphi(s) \text { on }-\delta \leq s \leq 0, \quad \theta(1)=\gamma
$$

After quasilinearization, we have a sequence $\left\langle\theta^{(v)}\right\rangle$ of linear equations defined by the following recurrence 
relation:

$$
\begin{gathered}
\varepsilon \theta^{\prime \prime(v+1)}(s)=\mathscr{F}\left(\theta^{(v)}\right)+\left(\theta^{(v+1)}(s)-\theta^{(v)}(s)\right) \mathscr{F}^{\prime}\left(\theta^{(v)}\right), \\
\theta^{(v+1)}(s)=\varphi(s),-\delta \leq s \leq 0, \theta^{(v+1)}(1)=\gamma,
\end{gathered}
$$

where $\mathscr{F}^{\prime}(\theta)=\partial \mathscr{F}(\theta) / \partial \theta$.

Let $\theta^{(0)}(s)$ be an initial approximation; then using Equation (12), we have

$$
\varepsilon \theta^{\prime \prime(v)}(s)=\mathscr{F}\left(\theta^{(v-1)}\right)+\left(\theta^{(v)}(s)-\theta^{(v-1)}(s)\right) \mathscr{F}^{\prime}\left(\theta^{(v-1)}\right) .
$$

Using Equations (12) and (14), we have

$$
\begin{aligned}
\varepsilon\left(\theta^{(v+1)}(s)-\theta^{(v)}(s)\right)^{\prime \prime}= & \mathscr{F}\left(\theta^{(v)}\right)-\mathscr{F}\left(\theta^{(v-1)}\right)-\left(\theta^{(v)}(s)-\theta^{(v-1)}(s)\right) \mathscr{F}^{\prime}\left(\theta^{(v-1)}\right) \\
& +\left(\theta^{(v+1)}(s)-\theta^{(v)}(s)\right) \mathscr{F}^{\prime}\left(\theta^{(v)}\right) .
\end{aligned}
$$

Equation (15) is a differential equation of second order in $\left(\theta^{(v+1)}(s)-\theta^{(v)}(s)\right)$. Thus, by using Green's function, the integral form of Equation (15) is

$$
\begin{aligned}
\varepsilon\left(\theta^{(v+1)}(s)-\theta^{(v)}(s)\right)= & \int_{0}^{1} G(s, t)\left[\mathscr{F}\left(\theta^{(v)}\right)-\mathscr{F}\left(\theta^{(v-1)}\right)-\left(\theta^{(v)}(s)-\theta^{(v-1)}(s)\right) \mathscr{F}^{\prime}\left(\theta^{(v-1)}\right)\right. \\
& \left.+\left(\theta^{(v+1)}(s)-\theta^{(v)}(s)\right) \mathscr{F}^{\prime}\left(\theta^{(v)}\right)\right] d s,
\end{aligned}
$$

where the $G(s, t)$ is the Green's function and determined by [21]

$$
G(s, t)= \begin{cases}(s-1) t, & 0 \leq t \leq s \leq 1 \\ s(t-1), & 0 \leq s \leq t \leq 1\end{cases}
$$

where $\max _{s, t}|G(s, t)|=1 / 4$. By using the mean value theorem, we have

$$
\begin{aligned}
\mathscr{F}\left(\theta^{(v)}\right)-\mathscr{F}\left(\theta^{(v-1)}\right)= & \left(\theta^{(v)}(s)-\theta^{(v-1)}(s)\right) \mathscr{F}^{\prime}\left(\theta^{(v-1)}\right) \\
& +\frac{\left(\theta^{(v)}(s)-\theta^{(v-1)}(s)\right)^{2}}{2} \mathscr{F}^{\prime \prime}(t),
\end{aligned}
$$

where $\theta^{(v-1)}(s) \leq t \leq \theta^{(v)}(s)$. Substituting Equation (18) into Equation (16), we get

$$
\begin{aligned}
\varepsilon\left(\theta^{(v+1)}(s)-\theta^{(v)}(s)\right)= & \int_{0}^{1} G(s, t)\left[\left(\mathscr{F}\left(\theta^{(v)}\right)-\mathscr{F}\left(\theta^{(v-1)}\right)\right)^{2} \frac{\mathscr{F}^{\prime \prime}(\theta)}{2}\right. \\
& \left.+\left(\theta^{(v+1)}(s)-\theta^{(v)}(s)\right) \mathscr{F}^{\prime}\left(\theta^{(v)}\right)\right] d s .
\end{aligned}
$$

Let $\max _{\|w\| \leq 1} \mathscr{F}^{\prime}(\theta)=a_{1}, \max _{\|w\| \leq 1} \mathscr{F}^{\prime \prime}(\theta)=a_{2}$. On both sides of Equation (18), taking the maximum of the moduli over the region of interest, we get

$$
\begin{aligned}
\max _{0 \leq s \leq 1}\left|\theta^{(v+1)}(s)-\theta^{(v)}(s)\right| \leq & \frac{1}{4 \varepsilon} \int_{0}^{1}\left[\max _{0 \leq s \leq 1} \frac{\left(\theta^{(v)}(s)-\theta^{(v-1)}(s)\right)}{2} \max _{0 \leq s \leq 1}\left|\mathscr{F}^{\prime \prime}(\theta)\right|\right] d s \\
& +\frac{1}{4 \varepsilon} \int_{0}^{1}\left[\max _{0 \leq s \leq 1}\left|\left(\theta^{(v)}(s)-\theta^{(v-1)}(s)\right)\right| \max _{0 \leq s \leq 1}\left|\mathscr{F}^{\prime}\left(\theta^{(v)}\right)\right|\right] d s .
\end{aligned}
$$

A simplification yields

$$
\max _{0 \leq s \leq 1}\left|\theta^{(v+1)}(s)-\theta^{(v)}(s)\right| \leq K_{1} \max _{0 \leq s \leq 1}\left(\theta^{(v)}(s)-\theta^{(v-1)}(s)\right)^{2},
$$

where $K_{1}=\left(a_{2} /\left(8 \varepsilon\left(1-a_{1} / 4 \varepsilon\right)\right)\right)<1$. This shows that, given $K_{1}<1$, the sequence $\left\langle\theta^{(v)}(s)\right\rangle$ of linear equations converges quadratically. As a result, to get the approximate solution of Equation (1) with Equation (2), it is required to estimate the solution of the sequence of SPLDDEs of the form

$$
\varepsilon \theta^{\prime \prime(v+1)}(s)+a^{v}(s) \theta^{\prime(v+1)}(s-\delta)+b^{v}(s) \theta^{(v+1)}(s)=c^{v}(s), \quad v=0,1, \cdots,
$$

with

$$
\theta^{(v+1)}(s)=\mu(s),-\delta \leq s \leq 0, \quad \theta^{(v+1)}(1)=\gamma,
$$

where

$$
\begin{gathered}
a^{v}(s)=\left(-\frac{\partial \mathscr{F}^{(v)}}{\partial \theta^{\prime}}\right), \\
b^{v}(s)=\left(-\frac{\partial \mathscr{F}^{v}}{\partial \theta}\right), \\
c^{v}(s)=\left(\mathscr{F}^{v}-\theta^{(v)}\left(\frac{\partial \mathscr{F}^{(v)}}{\partial \theta}\right)-\left(\frac{\partial \mathscr{F}^{(v)}}{\partial \theta^{\prime}}\right) \theta^{\prime(v)}(s-\delta)\right) .
\end{gathered}
$$

\section{Continuous Problem}

When the delay argument $\delta$ is $o(\varepsilon)$, sequential expanding for the term $\theta^{\prime(v+1)}(s-\delta)$ in Equation (22) yields

$$
L_{\pi}=\varepsilon \theta^{\prime \prime(v+1)}(s)+p^{v}(s) \theta^{\prime(v+1)}(s)+q^{v}(s) \theta^{(v+1)}(s)=r^{v}(s), v=0,1, \cdots,
$$


where

$$
\begin{gathered}
p^{v}(s)=\frac{a^{v}(s)}{\left(1-\left(a^{v}(s) \delta / \varepsilon\right)\right)}, \\
q^{n}(s)=\frac{b^{v}(s)}{\left(1-\left(b^{v}(s) \delta / \varepsilon\right)\right)}, \\
r^{v}(s)=\frac{\mathscr{F}^{v}(s)-\theta^{(v)}\left(\partial \mathscr{F}^{(v)} / \partial \theta\right)-\left[\theta^{\prime(v)}(s)-\delta \theta^{\prime \prime(v)}(s)\right]\left(\partial \mathscr{F}^{(v)} / \partial \theta^{\prime}\right)}{\left(1-\left(a^{v}(s) \delta / \varepsilon\right)\right)},
\end{gathered}
$$

with

$$
\theta^{(v+1)}(0)=\mu(0)=\mu_{0}, \theta^{(v+1)}(1)=\gamma .
$$

The boundary layer appears on the left or right side of the interval depending on the sign of the coefficient $p^{v}(s)$, i.e., as $p^{v}(s)>0$ or $p^{v}(s)<0$, respectively.

The operator $L_{\pi}=\varepsilon\left(d^{2} / d s^{2}\right)+p^{v}(s)(d / d s)+q^{v}(s) I$ in Equation (25) satisfies Lemma 1.

Lemma 1. Assume $\mathscr{G}(s)$ is a smooth function satisfying $\mathscr{G}^{(v+1)}(0) \geq 0, \mathscr{G}^{(v+1)}(1) \geq 0$. Then, $L_{\pi} \mathscr{G}^{(v+1)}(s) \leq 0, \forall x \in(0$, 1) implies $\mathscr{G}^{(v+1)}(s) \geq 0, \forall s \in[0,1], k=0,1, \cdots$

Proof. Let $k \in[0,1]$ be such that $\mathscr{G}^{(v+1)}(k)<0$ and

$$
\mathscr{G}^{(v+1)}(k)=\min _{s \in[0,1]} \mathscr{G}^{(v+1)}(s)
$$

Clearly, $k \notin\{0,1\}$; therefore, $\mathscr{G}^{(v+1)}(k)=0$ and $\mathscr{G}^{\prime \prime^{(v+1)}}$ $(k) \geq 0$.

Now, consider

$\left.L_{\pi} \mathscr{G}^{(v+1)}(m)\right|_{s=k}=\varepsilon \mathscr{G}^{\prime{ }^{\prime(v+1)}}(k)+p^{v}(k) \mathscr{G}^{\prime(v+1)}(k)+q^{v}(k) \mathscr{G}^{(v+1)}(k)>0$,

which is opposite to our assumption.

Hence, $\mathscr{G}^{(v+1)}(s) \geq 0, \forall s \in[0,1]$.

Lemma 2. Let $\theta^{(v+1)}(s)$ be the solution of Equation (25) with Equation (27); then, we have

$$
\left\|\theta^{(v+1)}\right\| \leq\left(\beta^{*}\right)^{-1}\left\|r^{(v)}\right\|+\max \left(\left|\mu_{0}\right|,|\gamma|\right), \quad \forall v=0,1, \cdots,
$$

where $\|$.$\| is the L_{\infty}$ norm given by $\left\|\theta^{(v+1)}\right\|=\max _{0 \leq s \leq 1}\left|\theta^{(v+1)}(s)\right|$.
Proof. Let $\psi^{ \pm(v+1)}(s)$ be two barrier functions specified by

$$
\psi^{ \pm(v+1)}(s)=\left(\beta^{*}\right)^{-1}\left\|r^{(v)}\right\|+\max \left(\left|\mu_{0}\right|,|\gamma|\right) \pm \theta^{(v+1)}(s) .
$$

Then, this implies

$$
\begin{aligned}
\psi^{ \pm(v+1)}(s) & =\left(\beta^{*}\right)^{-1}\left\|r^{(v)}\right\|+\max \left(\left|\mu_{0}\right|,|\gamma|\right) \pm \theta^{(v+1)}(0) \\
& =\left(\beta^{*}\right)^{-1}\left\|r^{(v)}\right\|+\max \left(\left|\mu_{0}\right|,|\gamma|\right) \pm \mu_{0} \geq 0, \\
\psi^{ \pm(v+1)}(s) & =\left(\beta^{*}\right)^{-1}\left\|r^{(v)}\right\|+\max \left(\left|\mu_{0}\right|,|\gamma|\right) \pm \theta^{(v+1)}(1) \\
& =\left(\beta^{*}\right)^{-1}\left\|r^{(v)}\right\|+\max \left(\left|\mu_{0}\right|,|\gamma|\right) \pm \gamma \geq 0,
\end{aligned}
$$

$$
\begin{aligned}
\mathrm{L}_{\pi} \psi^{(v+1)}(s)= & \varepsilon\left(\psi^{ \pm(v+1)}(s)\right)^{\prime \prime}(s)+p^{v}(s)\left(\psi^{ \pm(v+1)}(s)\right)^{\prime} \\
& +q^{v}(s)\left(\psi^{ \pm(v+1)}(s)\right) \\
= & q^{(v)}(s)\left[\left(\beta^{*}\right)^{-1}\left\|r^{(v)}\right\|+\max \left(\left|\mu_{0}\right|,|\gamma|\right)\right] \\
& \pm L_{\pi} \theta^{(v+1)}(s) \\
= & q^{(v)}(s)\left[\left(\beta^{*}\right)^{-1}\left\|r^{(v)}\right\|+\max \left(\left|\mu_{0}\right|,|\gamma|\right)\right] \pm r^{(v)}(s) .
\end{aligned}
$$

As $q^{v}(s) \leq-\beta^{*}<0$ implies $q^{v}(s) \leq\left(\beta^{*}\right)^{-1} \leq-1$ and since $\left\|r^{(v)}\right\| \geq r^{(v)}(s)$, we have $L_{\pi} \phi^{(v+1)}(s) \leq 0, \forall s \in[0,1]$. Using Lemma 1 , the required estimate is obtained.

Lemma 3. If $\theta^{(v+1)}(s)$ satisfies Equations (25)-(27), then

$$
\theta^{(v+1)}(s)=u^{(v+1)}(s)+v^{(v+1)}(s)+z^{(v+1)}(s), \quad v=0,1,2, \cdots,
$$

where

$$
\begin{gathered}
u^{(v+1)}(s)=-\frac{\varepsilon \theta^{\prime(v+1)}(0)}{p^{(v)}(0)} \exp \left(\frac{-p^{(v)}(0) s}{\varepsilon}\right), \\
v^{(v+1)}(s)=-\frac{\varepsilon \theta^{\prime(v+1)}(1)}{p^{(v)}(1)} \exp \left(\frac{-p^{(v)}(1)(1-s)}{\varepsilon}\right), \\
z^{(v+1)}(s) \leq M\left\{1+\varepsilon^{-s+1} \exp \left(\frac{-c_{1}(1-s)}{\varepsilon}\right)\right\}, \quad s=0,1,2,3,4,5,6,
\end{gathered}
$$

where $c_{1}$ is constant and $M$ is positive constant independent of $h$ and $\varepsilon$.

\section{Trigonometric Spline}

The integration domain $[0,1]$ with mesh size $h=1 / N$ is decomposed into $N$ equal subintervals, so that $s_{i}=i h, i=0$ 
TABle 1: MAEs in Example 1 with $\delta=0.8 \varepsilon$.

\begin{tabular}{|c|c|c|c|c|c|c|}
\hline$\varepsilon / N$ & 64 & 128 & 256 & 512 & 1024 & 2048 \\
\hline \multicolumn{7}{|c|}{ Proposed method } \\
\hline $10^{-1}$ & $1.1651 e-03$ & $3.2884 e-04$ & $8.7186 e-05$ & $2.2435 e-05$ & $5.6893 e-06$ & $1.4325 e-06$ \\
\hline $10^{-2}$ & $3.3515 e-03$ & $1.0682 e-03$ & $2.9959 e-04$ & $7.9176 e-05$ & $2.0341 e-05$ & $5.1542 e-06$ \\
\hline $10^{-3}$ & $3.8819 e-03$ & $1.2730 e-03$ & $3.6165 e-04$ & $9.6155 e-05$ & $2.4775 e-05$ & $6.2866 e-06$ \\
\hline $10^{-4}$ & $3.9415 e-03$ & $1.2967 e-03$ & $3.6893 e-04$ & $9.8159 e-05$ & $2.5299 e-05$ & $6.4208 e-06$ \\
\hline $10^{-5}$ & $3.9476 e-03$ & $1.2991 e-03$ & $3.6967 e-04$ & $9.8363 e-05$ & $2.5352 e-05$ & $6.4344 e-06$ \\
\hline $10^{-6}$ & $3.9482 e-03$ & $1.2994 e-03$ & $3.6974 e-04$ & $9.8383 e-05$ & $2.5358 e-05$ & $6.4358 e-06$ \\
\hline $10^{-7}$ & $3.9482 e-03$ & $1.2994 e-03$ & $3.6975 e-04$ & $9.8385 e-05$ & $2.5358 e-05$ & $6.4359 e-06$ \\
\hline $10^{-8}$ & $3.9482 e-03$ & $1.2994 e-03$ & $3.6975 e-04$ & $9.8385 e-05$ & $2.5358 e-05$ & $6.4359 e-06$ \\
\hline \multicolumn{7}{|c|}{ Results in [15] } \\
\hline $10^{-1}$ & $6.036 e-02$ & $1.973 E-02$ & $5.134 e-03$ & $1.276 e-03$ & $3.185 e-04$ & $7.963 e-05$ \\
\hline $10^{-2}$ & $5.894 e-02$ & $1.902 e-02$ & $5.945 e-03$ & $1.812 e-03$ & $5.279 e-04$ & $1.444 e-04$ \\
\hline $10^{-3}$ & $5.891 e-02$ & $1.895 e-02$ & $5.906 e-03$ & $1.791 e-03$ & $5.164 e-04$ & $1.382 e-04$ \\
\hline $10^{-4}$ & $5.893 e-02$ & $1.902 e-02$ & $5.921 e-03$ & $1.789 e-03$ & $5.153 e-04$ & $1.376 e-04$ \\
\hline $10^{-5}$ & $5.892 e-02$ & $1.901 e-02$ & $5.943 e-03$ & $1.806 e-03$ & $5.172 e-04$ & $1.376 e-04$ \\
\hline $10^{-6}$ & $5.899 e-02$ & $1.901 e-02$ & $5.938 e-03$ & $1.806 e-03$ & $5.250 e-04$ & $1.409 e-04$ \\
\hline $10^{-7}$ & $5.973 e-02$ & $1.902 e-02$ & $5.938 e-03$ & $1.805 e-03$ & $5.237 e-04$ & $1.420 e-04$ \\
\hline $10^{-8}$ & $6.713 e-02$ & $1.915 e-02$ & $5.940 e-03$ & $1.805 e-03$ & $5.234 e-04$ & $1.416 e-04$ \\
\hline
\end{tabular}

TABle 2: MAEs in Example 1 with $\delta=0.5 \varepsilon$.

\begin{tabular}{|c|c|c|c|c|c|}
\hline$\varepsilon / N$ & 32 & 64 & 128 & 256 & 512 \\
\hline \multicolumn{6}{|c|}{ Proposed method } \\
\hline $2^{-1}$ & $3.6533 e-04$ & $9.5648 e-05$ & $2.4452 e-05$ & $6.1804 e-06$ & $1.5535 e-06$ \\
\hline $2^{-2}$ & $5.6399 e-04$ & $1.4855 e-04$ & $3.8081 e-05$ & $9.6382 e-06$ & $2.4243 e-06$ \\
\hline $2^{-4}$ & $9.0467 e-04$ & $2.4051 e-04$ & $6.1920 e-05$ & $1.5704 e-05$ & $3.9539 e-06$ \\
\hline $2^{-6}$ & $1.0520 e-03$ & $2.8073 e-04$ & $7.2400 e-05$ & $1.8377 e-05$ & $4.6288 e-06$ \\
\hline $2^{-8}$ & $1.0954 e-03$ & $2.9264 e-04$ & $7.5510 e-05$ & $1.9171 e-05$ & $4.8293 e-06$ \\
\hline $2^{-12}$ & $1.1097 e-03$ & $2.9655 e-04$ & $7.6529 e-05$ & $1.9431 e-05$ & $4.8950 e-06$ \\
\hline $2^{-16}$ & $1.1106 e-03$ & $2.9679 e-04$ & $7.6594 e-05$ & $1.9447 e-05$ & $4.8992 e-06$ \\
\hline $2^{-32}$ & $1.1106 e-03$ & $2.9681 e-04$ & $7.6598 e-05$ & $1.9449 e-05$ & $4.8995 e-06$ \\
\hline \multicolumn{6}{|c|}{ Result in [23] } \\
\hline $2^{-1}$ & $4.84 e-4$ & $1.31 e-4$ & $3.39 e-5$ & $8.66 e-6$ & $2.18 e-6$ \\
\hline $2^{-2}$ & $1.54 e-3$ & $4.28 e-4$ & $1.13 e-4$ & $2.90 e-5$ & $7.37 e-6$ \\
\hline $2^{-4}$ & $8.86 e-3$ & $2.82 e-3$ & $8.17 e-4$ & $2.21 e-4$ & $5.78 e-5$ \\
\hline $2^{-6}$ & $3.87 e-2$ & $1.23 e-3$ & $2.91 e-3$ & $6.93 e-4$ & $1.68 e-4$ \\
\hline $2^{-8}$ & $3.68 e-2$ & $8.95 e-3$ & $2.12 e-3$ & $5.22 e-4$ & $1.30 e-4$ \\
\hline $2^{-12}$ & $4.05 e-2$ & $8.33 e-3$ & $1.87 e-3$ & $4.46 e-4$ & $1.11 e-4$ \\
\hline $2^{-16}$ & $4.84 e-2$ & $8.33 e-3$ & $1.87 e-3$ & $4.45 e-4$ & $1.09 e-4$ \\
\hline $2^{-32}$ & $4.84 e-2$ & $8.33 e-3$ & $1.87 e-3$ & $4.45 e-4$ & $1.09 e-4$ \\
\hline
\end{tabular}


TABle 3: MAEs in Example 2 with $\delta=0.4 \varepsilon$.

\begin{tabular}{|c|c|c|c|c|c|c|}
\hline$\varepsilon / N$ & 64 & 128 & 256 & 512 & 1024 & 2048 \\
\hline \multicolumn{7}{|c|}{ Proposed method } \\
\hline $10^{-2}$ & $1.9998 e-04$ & $5.2245 e-05$ & $1.3345 e-$ & $3.3720 e-06$ & $8.4746 e-07$ & $2.1242 e-07$ \\
\hline $10^{-3}$ & $2.0404 e-04$ & $5.3327 e-05$ & $1.3624 e-$ & $3.4429 e-06$ & $8.6532 e-07$ & $2.1690 e-07$ \\
\hline $10^{-4}$ & $2.0446 e-04$ & $5.3438 e-05$ & $1.3653 e-$ & $3.4501 e-06$ & $8.6714 e-07$ & $2.1736 e-07$ \\
\hline $10^{-5}$ & $2.0450 e-04$ & $5.3449 e-05$ & $1.3656 e-$ & $3.4508 e-06$ & $8.6732 e-07$ & $2.1741 e-07$ \\
\hline $10^{-6}$ & $2.0450 e-04$ & $5.3450 e-05$ & $1.3656 e-$ & $3.4509 e-06$ & $8.6734 e-07$ & $2.1741 e-07$ \\
\hline $10^{-7}$ & $2.0450 e-04$ & $5.3450 e-05$ & $1.3656 e-$ & $3.4509 e-06$ & $8.6734 e-07$ & $2.1741 e-07$ \\
\hline $10^{-8}$ & $2.0450 e-04$ & $5.3450 e-05$ & $1.3656 e-$ & $3.4509 e-06$ & $8.6734 e-07$ & $2.1741 e-07$ \\
\hline \multicolumn{7}{|c|}{ Results in [15] } \\
\hline $10^{-2}$ & $5.623 e-02$ & $1.717 e-02$ & $5.387 e-03$ & $1.630 e-03$ & $4.750 e-04$ & $1.300 e-04$ \\
\hline $10^{-3}$ & $5.641 e-02$ & $1.718 e-02$ & $5.367 e-03$ & $1.620 e-03$ & $4.692 e-04$ & $1.269 e-04$ \\
\hline $10^{-4}$ & $5.640 e-02$ & $1.727 e-02$ & $5.418 e-03$ & $1.623 e-03$ & $4.687 e-04$ & $1.266 e-04$ \\
\hline $10^{-5}$ & $5.638 e-02$ & $1.725 e-02$ & $5.426 e-03$ & $1.650 e-03$ & $4.776 e-04$ & $1.267 e-04$ \\
\hline $10^{-6}$ & $5.638 e-02$ & $1.725 e-02$ & $5.421 e-03$ & $1.646 e-03$ & $4.836 e-04$ & $1.338 e-04$ \\
\hline $10^{-7}$ & $5.640 e-02$ & $1.725 e-02$ & $5.420 e-03$ & $1.645 e-03$ & $4.820 e-04$ & $1.335 e-04$ \\
\hline $10^{-8}$ & $5.655 e-02$ & $1.725 e-02$ & $5.420 e-03$ & $1.645 e-03$ & $4.817 e-04$ & $1.331 e-04$ \\
\hline
\end{tabular}

TABle 4: MAEs in Example 2 with $\delta=0.5 \varepsilon$.

\begin{tabular}{|c|c|c|c|c|c|}
\hline$\varepsilon / N$ & 32 & 64 & 128 & 256 & 512 \\
\hline \multicolumn{6}{|c|}{ Proposed method } \\
\hline $2^{-1}$ & $2.1589 e-06$ & $9.4190 e-07$ & $2.3548 e-07$ & $5.8869 e-08$ & $1.4717 e-08$ \\
\hline $2^{-2}$ & $2.2793 e-05$ & $4.9244 e-06$ & $1.2344 e-06$ & $3.0893 e-07$ & $5.0693 e-08$ \\
\hline $2^{-4}$ & $2.1081 e-05$ & $1.2064 e-06$ & $3.3601 e-06$ & $2.9526 e-07$ & $5.3741 e-08$ \\
\hline $2^{-8}$ & $2.7909 e-05$ & $7.0930 e-06$ & $1.7870 e-06$ & $3.0396 e-07$ & $7.5799 e-08$ \\
\hline $2^{-12}$ & $2.7908 e-05$ & $7.0926 e-06$ & $1.7869 e-06$ & $3.0461 e-07$ & $7.5949 e-08$ \\
\hline $2^{-16}$ & $2.7908 e-05$ & $7.0926 e-06$ & $1.7869 e-06$ & $3.0461 e-07$ & $7.5949 e-08$ \\
\hline $2^{-18}$ & $2.7908 e-05$ & $7.0926 e-06$ & $1.7869 e-06$ & $3.0461 e-07$ & $7.5949 e-08$ \\
\hline \multicolumn{6}{|c|}{ Result in [23] } \\
\hline $2^{-1}$ & $7.32 e-6$ & $1.71 e-6$ & $4.07 e-7$ & $9.85 e-8$ & $2.42 e-8$ \\
\hline $2^{-2}$ & $2.88 e-5$ & $6.10 e-6$ & $1.37 e-6$ & $3.21 e-7$ & $7.75 e-8$ \\
\hline $2^{-4}$ & $4.98 e-5$ & $9.33 e-6$ & $1.92 e-6$ & $4.26 e-7$ & $9.96 e-8$ \\
\hline $2^{-8}$ & $2.34 e-4$ & $4.54 e-5$ & $6.89 e-6$ & $9.22 e-7$ & $1.20 e-7$ \\
\hline $2^{-12}$ & $2.41 e-4$ & $5.85 e-5$ & $1.43 e-5$ & $3.54 e-6$ & $8.48 e-7$ \\
\hline $2^{-16}$ & $2.41 e-4$ & $5.85 e-5$ & $1.43 e-5$ & $3.54 e-6$ & $8.80 e-7$ \\
\hline $2^{-18}$ & $2.41 e-4$ & $5.85 e-5$ & $1.43 e-5$ & $3.54 e-6$ & $8.80 e-7$ \\
\hline
\end{tabular}

$, 1,2, \cdots, N$ are the nodes with $0=s_{0}, 1=s_{N}$. Let $\theta(s)$ be the exact solution and $\theta_{i}$ be an approximation to $\theta\left(s_{i}\right)$ by the trigonometric spline $S_{i}(s)$ passing through the points $\left(s_{i}, \theta_{i}\right.$ ) and $\left(s_{i+1}, \theta_{i+1}\right)$. Here, $S_{i}(s)$ meets the interpolatory conditions at $s_{i}$ and $s_{i+1}$; also the continuity of first derivatives at the common nodes $\left(s_{i}, \theta_{i}\right)$ is fulfilled. For each $i^{\text {th }}$ subinter- val, the trigonometric spline function $S_{i}(s)$ has the form

$S_{i}(s)=a_{i}+b_{i}\left(s-s_{i}\right)+c_{i} \sin \tau\left(s-s_{i}\right)+d_{i} \cos \tau\left(s-s_{i}\right), \quad i=0,1, \cdots, N-1$,

where $a_{i}, b_{i}, c_{i}$, and $d_{i}$ are constants and $\tau$ is a free parameter. 


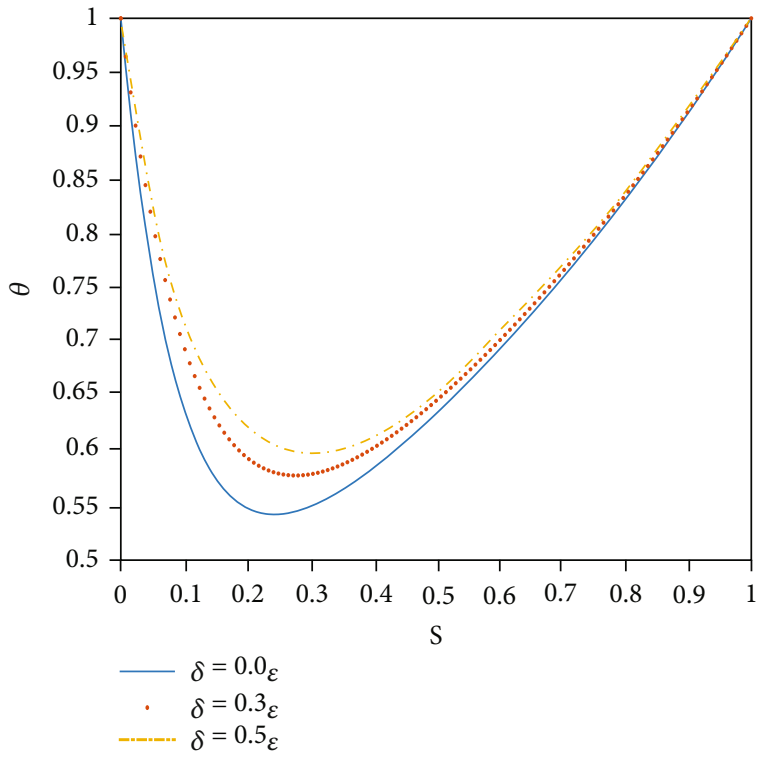

FIgURe 1: Layer profile in Example 1 with $\varepsilon=10^{-1}$.

To get the values of the coefficients of Equation (35) in terms of $\theta_{i}, \theta_{i+1}, M_{i}$, and $M_{i+1}$, the following are defined:

$$
S\left(s_{i}\right)=\theta_{i}, S\left(s_{i+1}\right)=\theta_{i+1}, s^{\prime \prime}\left(s_{i}\right)=M_{i}, s^{\prime \prime}\left(s_{i+1}\right)=M_{i+1} .
$$

Using simple calculations, the following expressions are obtained for the coefficients:

$$
\begin{gathered}
a_{i}=\theta_{i}+\frac{M_{i}}{\tau^{2}}, \\
b_{i}=\frac{\theta_{i}-\theta_{i+1}}{h}+\frac{M_{i+1}-M_{i}}{\tau \theta}, \\
c_{i}=\frac{M_{i} \cos \theta-M_{i+1}}{\tau^{2} \sin \theta}, \\
d_{i}=-\frac{M_{i}}{\tau^{2}},
\end{gathered}
$$

where $\theta=\tau h$, for $i=0,1,2, \cdots, N-1$. Using the first derivative continuity at $\left(s_{i}, \theta_{i}\right)$, that is $S_{i+1}^{\prime}\left(s_{i}\right)=S_{i}^{\prime}\left(s_{i}\right)$, we get the following relation:

$\alpha M_{i+1}+2 \beta M_{i}+\alpha M_{i-1}=\frac{\theta_{i-1}-2 \theta_{i}+\theta_{i+1}}{h^{2}}, \quad$ for $i=1,2,3, \cdots, N-1$.

Here, $\alpha=\left(-1 / \theta^{2}\right)+(1 / \theta \sin \theta), \beta=\left(1 / \theta^{2}\right)-(\cos \theta / \theta \sin$ $\theta), M_{j}=\theta^{\prime \prime}\left(s_{j}\right), j=i-1, i . i+1$ and $\theta=\tau h$.

\section{Method of Solution}

At the grid points $s_{i}$, Equation (25) may be discretised by

$$
\varepsilon M_{j}^{(v+1)}=r^{v}\left(s_{j}\right)-p^{v}\left(s_{j}\right) \theta^{\prime(v+1)}\left(s_{j}\right)-q^{v}\left(s_{j}\right) \theta^{(v+1)}\left(s_{j}\right), \quad \forall j=i, i \pm 1 .
$$



Figure 2: Layer profile in Example 1 with $\varepsilon=10^{-2}$.

Using Equation (39) in Equation (38) and utilising the first derivatives of $\theta$ using the following estimations:

$$
\begin{gathered}
\theta_{i+1}^{\prime}(v+1)=\frac{\theta_{i-1}-4 \theta_{i}+3 \theta_{i+1}}{2 h}, \\
\theta_{i-1}^{\prime}(v+1)=\frac{-3 \theta_{i+1}+4 \theta_{i}-\theta_{i-1}}{2 h}, \\
\theta_{i}^{\prime}(v+1)=\left[\frac{1+2 \omega h^{2} q_{i+1}+\omega h\left[3 p_{i+1}+p_{i-1}\right]}{2 h}\right] \theta_{i+1} \\
-2 \omega\left[p_{i+1}+p_{i-1}\right] \theta_{i}-\left[\frac{1+2 \omega h^{2} q_{i-1}-\omega h\left[p_{i+1}+3 p_{i-1}\right]}{2 h}\right] \theta_{i-1} \\
+\omega h\left[r_{i+1}-r_{i-1}\right],
\end{gathered}
$$

we get

$$
\begin{aligned}
\{\varepsilon+ & \left.\frac{3 \alpha p_{i-1} h}{2}-\beta p_{i} w h^{2}\left[p_{i+1}+3 p_{i-1}\right]+2 \beta p_{i} \omega h^{3} q_{i-1}-\frac{\alpha h p_{i+1}}{2}-h^{2} \alpha q_{i-1}+h \beta p_{i}\right\} \theta_{i-1} \\
& +\left\{-2 \varepsilon-2 h \alpha p_{i-1}+4 \beta p_{i}\left[2 \omega\left(p_{i+1}+p_{i-1}\right)\right]+2 h \alpha p_{i+1}-2 h^{2} \beta q_{i}\right\} \theta_{i} \\
& +\left\{\varepsilon+\frac{\alpha h p_{i-1}}{2}-\beta p_{i} h^{2} \omega\left[3 p_{i+1}+p_{i-1}\right]-2 \beta p_{i} h^{3} \omega q_{i+1}-\frac{3 h \alpha p_{i+1}}{2}-h^{2} \alpha q_{i+1}-h \beta p_{i}\right\} \theta_{i+1} \\
= & h^{2}\left\{(\alpha-2 \beta h \omega) r_{i-1}+2 \beta r_{i}+(\alpha+2 \beta h \omega) r_{i+1}\right\} .
\end{aligned}
$$

Using Equation (41), we have the following tridiagonal system:

$$
E_{i-1} \theta_{i-1}+F_{i} \theta_{i}+G_{i+1} \theta_{i+1}=H_{i}, \quad \text { for } i=1,2,3, \cdots, N-1 .
$$




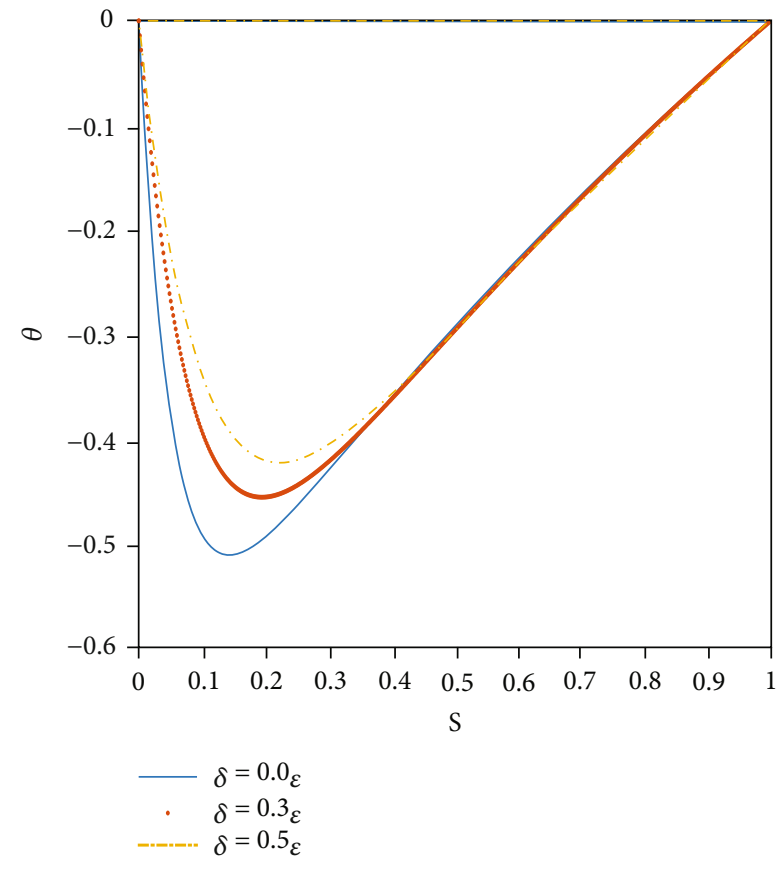

FIgURe 3: Layer profile in Example 2 with $\varepsilon=10^{-1}$.

Here,

$$
\begin{aligned}
E_{i-1}= & \varepsilon+\frac{3 \alpha p_{i-1} h}{2}-\beta p_{i} w h^{2}\left[p_{i+1}+3 p_{i-1}\right]+2 \beta p_{i} \omega h^{3} q_{i-1} \\
& -\frac{\alpha h p_{i+1}}{2}-h^{2} \alpha q_{i-1}+h \beta p_{i}
\end{aligned}
$$

$\mathrm{F}_{\mathrm{i}}=-2 \varepsilon-2 h \alpha p_{i-1}+4 \beta p_{i}\left[2 \omega\left(p_{i+1}+p_{i-1}\right)\right]+2 h \alpha p_{i+1}-2 h^{2} \beta q_{i}$

$$
\begin{aligned}
G_{i+1}= & \varepsilon+\frac{\alpha h p_{i-1}}{2}-\beta p_{i} h^{2} \omega\left[3 p_{i+1}+p_{i-1}\right]-2 \beta p_{i} h^{3} \omega q_{i+1} \\
& -\frac{3 h \alpha p_{i+1}}{2}-h^{2} \alpha q_{i+1}-h \beta p_{i}
\end{aligned}
$$$$
H_{i}=h^{2}\left\{(\alpha-2 \beta h \omega) r_{i-1}+2 \beta r_{i}+(\alpha+2 \beta h \omega) r_{i+1}\right\}
$$

\section{Error Estimate}

The truncation error in the proposed numerical scheme is given by

$$
\begin{aligned}
\mu_{i}(h)= & {[1-2(\alpha+\beta)] h^{2} \varepsilon \theta_{i}^{\prime \prime}+\left\{\left[\left(4 \omega \varepsilon+\frac{1}{3}\right) \beta-\frac{2 \alpha}{3}\right] P_{i} \theta_{i}^{\prime \prime \prime}\right.} \\
& \left.+(-1-12 \alpha) \frac{\varepsilon \theta_{i}^{4}}{12}\right\} h^{4}+o\left(h^{6}\right) .
\end{aligned}
$$

Thus, for different values of $\omega, \alpha$ and $\beta$ in the approach (Equation (42)), the following different orders are indicated:

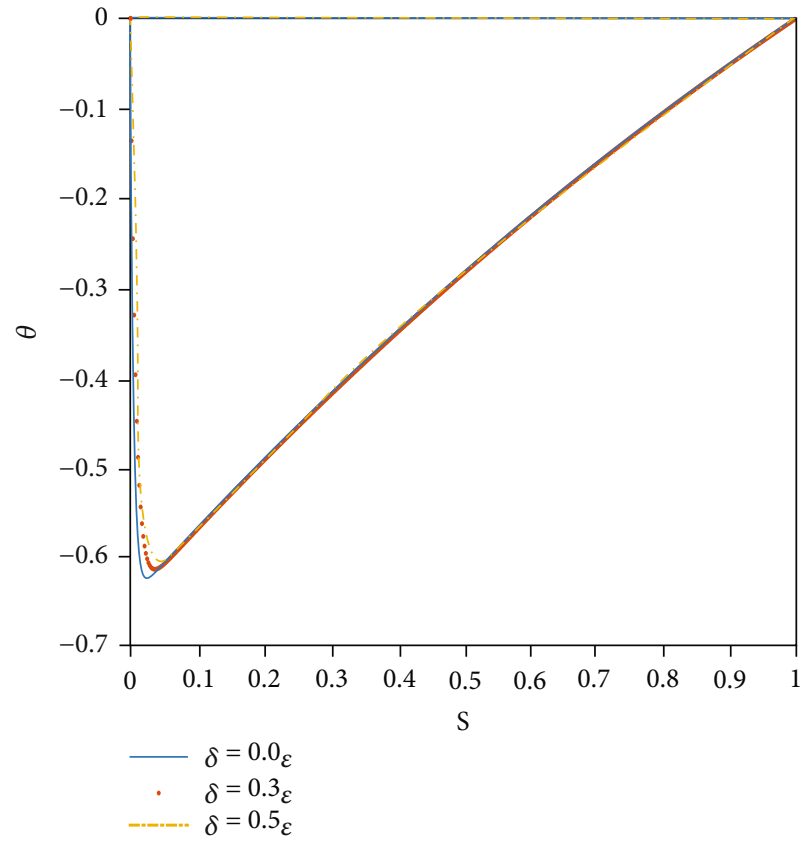

Figure 4: Layer profile in Example 2 with $\varepsilon=10^{-2}$.

(1) The scheme of Equation (44) yields the second order approach for any arbitrary choice of $\alpha$ and $\beta$ with $\alpha+\beta=1 / 2$ and for any value of $\omega$

(2) For $\alpha=1 / 12, \beta=5 / 12, \omega=-1 / 20 \varepsilon$, the scheme Equation (44) gives the sixth order

Theorem 4. Let $W_{j}^{(v+1)}, j=0,1,2, \cdots . N$ be an approximate solution to $\theta^{(v+1)}(s)$ of Equation (1) obtained by the proposed method. Then, the required estimate is

$$
\max _{j}\left|\theta_{j}^{(v+1)}-W_{j}^{(v+1)}\right| \leq M h^{4}\left[1+e^{\left(-k_{1} s_{j} / \varepsilon\right)}+e^{\left(-k_{2}\left(1-s_{j}\right) / \varepsilon\right)}\right],
$$

for $\alpha=1 / 12, \beta=5 / 12, \omega=-1 / 20 \varepsilon$. Here, $k_{1}, k_{2}, M$ are positive constants, independent of $h$ and $\varepsilon$.

Proof. Using Lemma 3, we have

$$
U_{j}^{(6)(v+1)}=\left(\frac{-p^{(v)}(0)}{\varepsilon}\right)^{5} \exp \left(\frac{-p^{(v)}(0) s_{j}}{\varepsilon}\right) .
$$

Therefore

$$
\left|\mu_{j}\left(u^{(v+1)}\right)\right| \leq \frac{M h^{6}}{\varepsilon^{5}} \exp \left(\frac{-p^{(v)}(0) s_{j}}{\varepsilon}\right) .
$$


Similarly,

$$
\begin{gathered}
\left|\mu_{j}\left(\vartheta^{(v+1)}\right)\right| \leq \frac{M h^{6}}{\varepsilon^{5}} \exp \left(\frac{-p^{(v)}(1)\left(1-s_{j}\right)}{\varepsilon}\right), \\
\left|z_{j}^{(6)(v+1)}\right| \leq M\left\{1+\varepsilon^{-5} \exp \left(\frac{-c_{1}\left(1-s_{j}\right)}{\varepsilon}\right)\right\}, \\
\left|\mu_{i}\left(z^{(v+1)}\right)\right| \leq M h^{6}\left\{1+\varepsilon^{-5} \exp \left(\frac{-c_{1}\left(1-s_{j}\right)}{\varepsilon}\right)\right\} .
\end{gathered}
$$

Now,

$$
\begin{gathered}
\mu_{i}\left(\theta^{(v+1)}\right)=\mu_{i}\left(u^{(k+1)}\right)+\mu_{i}\left(9^{(v+1)}\right)+\mu_{i}\left(z^{(v+1)}\right) \\
\left|\mu_{i}\left(\theta^{(v+1)}\right)\right| \leq \frac{M h^{6}}{\varepsilon^{5}}\left[1+\exp \left(\frac{-p^{(v)}(0) s_{j}}{\varepsilon}\right)+\exp \left(\frac{-p^{(v)}(1)\left(1-s_{j}\right)}{\varepsilon}\right)\right] .
\end{gathered}
$$

The matrix form of the system Equation (42) is

$$
A \theta^{(v+1)}=B, l=0,1, \cdots
$$

where $A$ is the matrix of the system Equation (42), $\theta^{(v+1)}$ and $B$ are the corresponding vectors, and $\mu_{i}\left(\theta^{(v+1)}\right)$ is the local truncation error. Thus,

$$
\max _{j}\left|\theta_{j}^{(v+1)}-W_{j}^{(v+1)}\right| \leq \| A^{-1}|| \max _{j}\left|\mu_{i}\left(\theta^{(v+1)}\right)\right|, \quad v=0,1, \cdots
$$

By using a result in [22], we have

$$
\left\|A^{-1}\right\| \leq \max _{1 \leq i \leq N-1}\left\{\left|E_{i}^{(v)}\right|+\left|F_{i}^{(v)}\right|+\left|G_{i}^{(v)}\right|\right\}, \quad v=0,1, \cdots,
$$

giving $\left\|A^{-1}\right\| \leq 1 / c h^{2}$.

Therefore,

$$
\max _{j}\left|\theta_{j}^{(v+1)}-W_{j}^{(v+1)}\right| \leq M h^{4}\left[1+e^{\left(-p^{(v)}(0) s_{j} / \varepsilon\right)}+e^{\left(\left(-p^{(v)}(1)\left(1-s_{j}\right)\right) / \varepsilon\right)}\right],
$$

for $\alpha=1 / 12, \beta=5 / 12, \omega=-1 / 20 \varepsilon$. Here, $k_{1}, k_{2}, M$ are positive constants, independent of $h$ and $\varepsilon$.

\section{Numerical Examples}

To show the relevance and validity of the approach, it was implemented for the following problems. The maximum pointwise errors (MAEs) $\left(E_{N, \varepsilon}^{K}\right)$ are determined by using the double mesh principle [3]:

$$
E_{N, \varepsilon}^{K}=\max _{0 \leq s \leq 1}\left|\left(\theta^{l}\right)_{i}^{N}-\left(\theta^{l}\right)_{2 i}^{2 N}\right| .
$$

Example 1. $\varepsilon \theta^{\prime \prime}(s)+\theta(s) \theta^{\prime}(s-\delta)-\theta(s)=0$ with the interval and boundary constraints

$$
\theta(\mathrm{s})=1,-\delta \leq s \leq 0, \theta(1)=1
$$

Example 2. $\varepsilon \theta^{\prime \prime}(s)+2 \theta^{\prime}(s-\delta)-e^{\theta(s)}=0$ with the interval and boundary constraints

$$
\theta(s)=0,-\delta \leq s \leq 0, \theta(1)=0
$$

\section{Conclusion}

To solve a singularly perturbed nonlinear delay differentiation equation, a computational technique is proposed using a trigonometric spline. The SPNDDE is reduced into a series of linear SPDDEs using quasilinearization. A trigonometric spline approach is suggested to solve the sequence of linear SPDDEs. The scheme was implemented on two problems. The values of the maximum absolute errors produced by the suggested scheme are compared to the results in [15, 23] presented in Tables 1-4. Comparisons reveal that the suggested scheme outperforms the methods given in [15, 23] in terms of maximum error. Results of simulation have shown that as we increase the value of the parameter $N$, the accuracy of the computed approximate solutions is significantly improved.

In addition, while the error values generally increase as the perturbation parameter $\varepsilon$ decreases, they are usually within reasonable limits even for small values of it. It is also worth noting that the approach works well even when $h \geq \varepsilon$ is used. Figures 1-4 depict the layer behaviour at various $\delta$ values. It has been noticed that when the delay value increases, the thickness of the boundary layer increases as well. The simulation results show that the computational method proposed in this study is capable of giving accurate results for SPNDDE.

\section{Data Availability}

The proposed equations usually play an important role in illustrating different applications, such as theory of nonpremixed combustion, geodynamics, oceanic and atmosphere circulation, and chemical reactions.

\section{Conflicts of Interest}

The authors declare that they have no conflicts of interest.

\section{References}

[1] F. A. Howes, Singular Perturbations and Differential Inequalities, American Mathematical Society, Providence, 1976. 
[2] K. W. Chang and F. A. Howes, Nonlinear Singular Perturbation Phenomena: Theory and Applications, Springer Science and Business Media, New York, 2012.

[3] T. C. Hanks, "Model relating heat-flow values near and vertical velocities of mass transport beneath, oceanic rises," Journal of Geophysical Research, vol. 76, pp. 537-544, 1971.

[4] G. F. Carrier, "Singular perturbation theory and geophysics," SIAM Review, vol. 12, pp. 175-193, 1970.

[5] R. Vulanovic, P. A. Farrell, and P. Lin, "Numerical solution of nonlinear singular perturbation problems modeling chemical reactions," in Applications of Advanced Computational Methods for Boundary and Interior Layers, pp. 192-213, Press publisher, 1993.

[6] E. P. Doolan, J. J. H. Miller, and W. H. A. Schilders, Uniform Numerical Methods for Problems with Initial and Boundary Layers, Advances in Numerical Computation Series, Boole Press Ltd., 1980.

[7] P. Narasimhan, Singularly Perturbed Delay Differential Equations and Numerical Methods, in Differential Equations and Numerical Analysis, Springer India, India, 2016.

[8] R. E. O’Malley, Introduction to Singular Perturbations, Academic Press, New York, 1974.

[9] S. Cengizci, "An asymptotic-numerical hybrid method for solving singularly perturbed linear delay differential equations," International Journal of Differential Equations, vol. 2017, 8 pages, 2017.

[10] C. G. Lange and R. M. Miura, "Singular perturbation analysis of boundary value problems for differential-difference equations. IV. A nonlinear example with layer behavior," Studies in Applied Mathematics, vol. 84, no. 3, pp. 231-273, 1991.

[11] Z. Bartoszewski and A. Baranowska, "Solving boundary value problems for second order singularly perturbed delay differential equations by $\varepsilon$-approximate fixed-point method," Mathematical Modelling and Analysis, vol. 20, pp. 369-381, 2015.

[12] M. Abdelhakema and Y. H. Youssri, "Two spectral Legendre's derivative algorithms for Lane-Emden, Bratu equations, and singular perturbed problems," Applied Numerical Mathematics, vol. 169, pp. 243-255, 2021.

[13] W. M. Abd-Elhameed, Y. H. Youssri, and E. H. Doha, "A novel operational matrix method based on shifted Legendre polynomials for solving second-order boundary value problems involving singular, singularly perturbed and Bratu-type equations," Mathematical Sciences, vol. 9, no. 2, pp. 93-102, 2015.

[14] M. K. Kadalbajoo and K. K. Sharma, "Numerical treatment for singularly perturbed nonlinear differential difference equations with negative shift," Non-linear Analysis: Theory, Methods and Applications, vol. 63, no. 5-7, pp. e1909-e1924, 2005.

[15] M. K. Kadalbajoo and D. Kumar, "A computational method for singularly perturbed nonlinear differential-difference equations with small shift," Applied Mathematical Modelling, vol. 34, pp. 2584-2596, 2010.

[16] A. H. Bhrawy, L. M. Assas, E. Tohidi, and M. A. Alghamdi, "A Legendre-Gauss collocation method for neutral functionaldifferential equations with proportional delays," Adv. Difference Equ., vol. 1, pp. 1-16, 2013.

[17] Y. Yang and E. Tohid, "Numerical solution of multipantograph delay boundary value problems via an efficient approach with the convergence analysis," Computational and Applied Mathematics, vol. 38, no. 3, pp. 1-14, 2019.
[18] S. Hosseinpour, A. Nazemi, and E. Tohidi, "A new approach for solving a class of delay fractional partial differential equations," Mediterranean Journal of Mathematics, vol. 15, no. 6, pp. 1-20, 2018.

[19] M. M. Kumar and A. S. V. Ravikanth, "A numerical approach for solving nonlinear singularly perturbed boundary value problem arising in control theory," Journal of Applied Nonlinear Dynamics, vol. 10, no. 1, pp. 151-159, 2021.

[20] R. E. Bellman and R. E. Kalaba, "Quasilinearization and nonlinear boundary-value problems," in Modern Analytic and Computational Methods in Science and Mathematics, American Elsevier Pub. Co., New York, 1965.

[21] I. Gohberg and S. Goldberg, Basic Operator Theory, Birkhäuser Publisher, 2013.

[22] J. M. Varah, "A lower bound for the smallest singular value of a matrix," Linear Algebra and its Applications, vol. 11, no. 1, pp. 3-5, 1975.

[23] A. S. V. Ravi Kanth and P. M. M. Kumar, "A numerical technique for solving nonlinear singularly perturbed delay differential equations," Mathematical Modelling and Analysis, vol. 23 , no. 1, pp. $64-78,2018$. 